\title{
Mean flow and spiral defect chaos in Rayleigh-Bénard convection
}

\author{
K.-H. Chiam,* M. R. Paul, and M. C. Cross \\ Nonlinear and Statistical Physics, Mail Code 114-36, California Institute of Technology, Pasadena, California 91125-3600 \\ H. S. Greenside \\ Department of Physics, P.O. Box 90305, Duke University, Durham, North Carolina 27708-0305
}

(Received 5 December 2002; published 14 May 2003)

\begin{abstract}
We describe a numerical procedure to construct a modified velocity field that does not have any mean flow. Using this procedure, we present two results. First, we show that, in the absence of the mean flow, spiral defect chaos collapses to a stationary pattern comprising textures of stripes with angular bends. The quenched patterns are characterized by mean wave numbers that approach those uniquely selected by focus-type singularities, which, in the absence of the mean flow, lie at the zigzag instability boundary. The quenched patterns also have larger correlation lengths and are comprised of rolls with less curvature. Secondly, we describe how the mean flow can contribute to the commonly observed phenomenon of rolls terminating perpendicularly into lateral walls. We show that, in the absence of the mean flow, rolls begin to terminate into lateral walls at an oblique angle. This obliqueness increases with the Rayleigh number.
\end{abstract}

DOI: 10.1103/PhysRevE.67.056206

PACS number(s): 47.54.+r, 47.27.Te, 47.52.+j

\section{INTRODUCTION}

Rayleigh-Bénard convection in a horizontal layer of fluid heated from below provides a canonical example for spatially extended systems exhibiting pattern formation [1] and spatiotemporal chaos [2-4]. (The latter term refers to states that are disordered in space and that show chaotic dynamics in time.) In this paper, we present results from direct numerical simulations of Rayleigh-Bénard convection to investigate the importance of nonlocal flow modes, generally referred to as the mean flow [5-9], in the formation and dynamics of patterns and spatiotemporal chaos.

In a typical Rayleigh-Bénard convection experiment, a fluid layer is confined between two horizontal plates, and is thermally driven far from equilibrium by maintaining the bottom plate at a temperature that is higher than that of the top plate. As the temperature difference is increased, the fluid undergoes an instability to a state in which there is motion driven by the buoyancy forces. When the temperature difference between the plates is above but near this convective threshold, a pattern comprised of patches of locally parallel convection rolls forms with roll diameters that are close to the depth of the cell; see, for example, Fig. 6(a). When the temperature difference is increased, the fluid undergoes other instabilities that may result in the pattern developing a simple or a chaotic time dependence. Finally, when the temperature difference is increased further, spatiotemporal chaotic states may appear. In particular, a state called spiral defect chaos [10] is observed for the Rayleigh number $R$ $\gtrsim 3000$, the Prandtl number $\sigma \sim 1$, and the aspect ratio $\Gamma$ $\gtrsim 16$. This state is a disordered collection of spirals that rotate in both directions and coexist with dynamical defects such as grain boundaries and dislocations [see, for example, Fig. 2(a)].

\footnotetext{
*Electronic address: ChiamKH@MailAPS.ORG

URL: http://www.cmp.caltech.edu/ stchaos
}

The following facts are known about spiral defect chaos: The parameter values for which it appears [10-14]; the distributions of local roll properties such as the wave number [15]; statistics of spiral and defect populations $[15,16]$; the mechanisms for the generation of chaos from spatial disorder [17]; the wave number selection mechanism for spirals $[18,19]$; and the conditions under which spiral defect chaos transitions to other states. Of particular interest to this paper are experiments $[20,21]$ that have observed that the spirals transform into targets when the Prandtl number is increased from $\sigma \sim 1$ to $\sigma \sim 10$ and when the Rayleigh number $R$ $\geq 3500$. While this observation establishes that spiral defect chaos occurs only at low Prandtl numbers, it does not allow us to conclude which of the many dynamic phenomena that occur at low Prandtl numbers [1] is responsible for the formation of spiral defect chaos.

One particular phenomenon that becomes important at low Prandtl numbers is the presence of a mean flow. Mean flow is the name given to the velocity field with a nonzero mean over the depth of the convective cell that is generated by the variations of the structure of the convection rolls such as their curvature, amplitude, and wave number, and that in turn couples through advection to further modify the roll structure. Its magnitude is approximately inversely proportional to the Prandtl number [22].

It is believed that spiral defect chaos is an effect of the mean flow which in turn is important at low Prandtl numbers $[10,14]$. This hypothesis has been investigated in numerical studies of model equations of convection. For example, by coupling a mean-flow-like field to the Swift-Hohenberg equation [23], chaotic behavior is observed [24]. Furthermore, when the parameter in the model that gives the strength of the mean flow is made large, spatiotemporal chaotic states akin to spiral defect chaos are observed [25-28]. However, the above results are tenuous because of two reasons. First, the Swift-Hohenberg equation describes the spatiotemporal behavior of a field in two dimensions, whereas convection is a three-dimensional phenomenon. Second, 


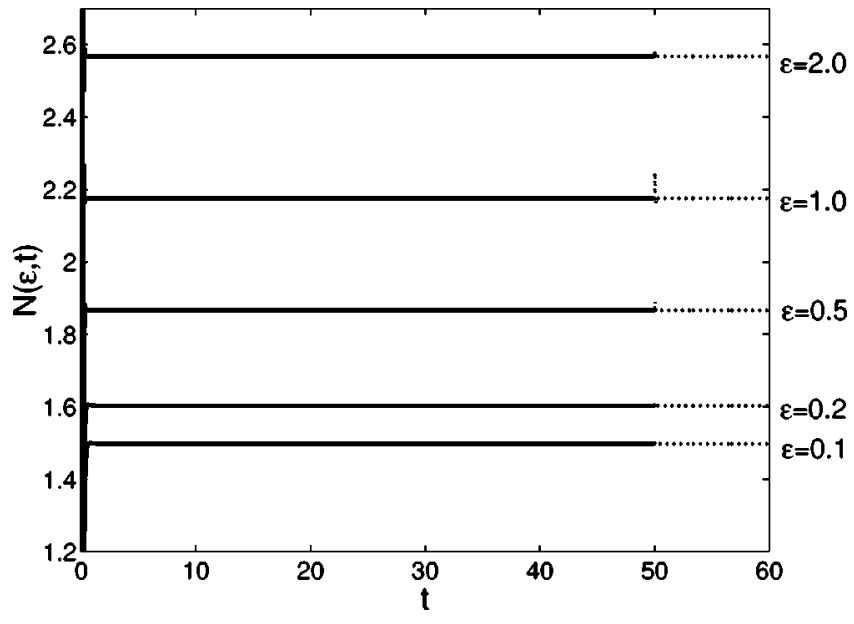

FIG. 1. Time series of the Nusselt number $N(\epsilon, t)$ for patterns comprising straight parallel rolls that have no mean flow at several values of $\epsilon$ before (denoted by solid lines) and after (dotted lines) quenching of the mean flow, which occurred at time $t_{q}=50$. All data reported here are for the Prandtl number $\sigma=1$ and a rectangular cell of aspect ratio $\Gamma_{x}=\Gamma_{y}=20$. (In order to achieve straight parallel rolls, periodic lateral boundaries were imposed and a small sinusoidal perturbation in the temperature field was used as the initial condition.)

there are known limitations to the Swift-Hohenberg modeling. For example, it is known that the long-time dynamics of the Swift-Hohenberg equation may not correspond to that of the Boussinesq equations of convection. In particular, SwiftHohenberg models exhibit spiral defect chaos as a transient behavior, whereas in experiments, spiral defect chaos is known to persist for much longer times [29]. In addition, it is also known that the small-scale structure of the mean flow at the cores of the spirals, which might be crucial for the persistence of spiral defect chaos, is not perfectly captured in the Swift-Hohenberg equation [29].

Our goal in this paper is to show by direct numerical simulations of Rayleigh-Bénard convection that spiral defect chaos is indeed a consequence of the presence of the mean flow. In the absence of the mean flow, we find that spiral defect chaotic states cease to exist, and are replaced by states whose statistical properties differ from those of spiral defect chaos. In general, studies of the mean flow are difficult to perform in experiments, primarily because it is difficult to measure the mean flow in an experimental setup [30-32]. This is due to several reasons, namely, that the magnitude of the mean flow is small (typically of the order of $1 \%$ of the magnitude of the velocity of the convecting rolls), and that it exists only in distorted and not regular patterns. To the best of our knowledge, there has only been one experiment that has successfully imaged aspects of the mean flow, but only in a simple distorted pattern $[33,34]$. It is not clear if such imaging techniques can be applied to more general and complicated patterns. Thus, direct numerical simulations are particularly valuable for the study of the mean flow.

We achieve this goal by numerically constructing a gedanken fluid whose velocity field is modified to have zero mean flow. By investigating the states that arise from the dynamics of this fluid and by comparing them with spiral (a)

(b)

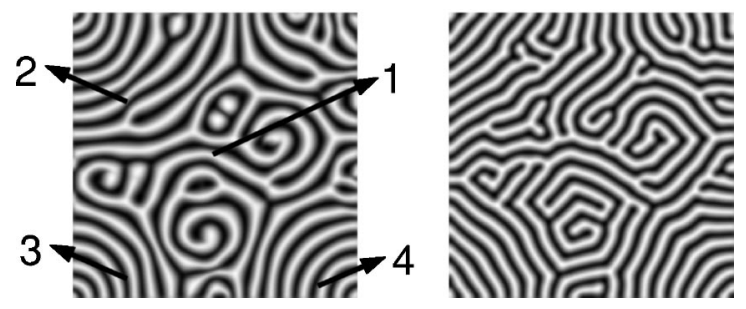

FIG. 2. (a) An example of spiral defect chaos observed in a numerical simulation using the finite-difference scheme [35]. The midplane temperature field is plotted at time $t=500$ for parameters $\epsilon=1.0, \sigma=1$, and $\Gamma_{x}=\Gamma_{y}=20$. Dark regions correspond to cold sinking fluid, light regions to hot rising fluid. The spiral defect chaos planform is characterized by a disordered collection of spirals rotating in both directions and coexisting with dynamical defects such as grain boundaries and dislocations. The labels " 1 " to " 4 " are discussed in Fig. 3. (b) When the mean flow is quenched, spiral defect chaos collapses to a stationary pattern of textures of stripes with angular bends. The planform shown here is at ten time units after the quenching has been introduced to the state shown in (a). All other parameters are unchanged.

defect chaos, we can infer directly the role of the mean flow in the formation and dynamics of spiral defect chaos.

Once we have the capability to remove the mean flow from the fluid dynamics, we can apply it to the study of other problems. One such problem that we have investigated is the relation between the mean flow and lateral boundaries. Using our numerical simulations, we have shown how the mean flow can contribute to the commonly observed phenomenon of convection rolls terminating perpendicularly into lateral walls, an observation that is still without much theoretical understanding.

The remaining part of this paper is organized as follows: In Sec. II, we define the equations governing RayleighBénard convection, what a mean flow is, and how it can be measured and eliminated numerically. In Sec. III, we present results on the relation between the mean flow and spiral defect chaos, stripe textures, and lateral boundaries. In Sec. IV, we present our conclusions.

\section{DEFINITIONS}

\section{A. Boussinesq equations}

The evolution of a low-velocity and hence approximately incompressible convecting fluid is governed to a good approximation by the three-dimensional Boussinesq equations [1]. They are the combination of the incompressible NavierStokes and heat equations, with the further assumption that density variations are proportional to temperature variations and that this density variation appears only in the buoyancy force. Written in a dimensionless form, they are

$$
\begin{gathered}
\sigma^{-1}\left(\partial_{t}+\mathbf{u} \cdot \boldsymbol{\nabla}\right) \mathbf{u}(x, y, z, t)=-\nabla p+\nabla^{2} \mathbf{u}+R T \hat{z}, \\
\left(\partial_{t}+\mathbf{u} \cdot \boldsymbol{\nabla}\right) T(x, y, z, t)=\nabla^{2} T
\end{gathered}
$$




$$
\boldsymbol{\nabla} \cdot \mathbf{u}=0 .
$$

The field $\mathbf{u}(x, y, z, t)$ is the velocity field at point $(x, y, z)$ at time $t$, while $p$ and $T$ are the pressure and temperature fields, respectively. The variables $x$ and $y$ denote the horizontal coordinates, while the variable $z$ denotes the vertical coordinate, with the unit vector $\hat{z}$ pointing in the direction opposite to the gravitational acceleration. The spatial units are measured in units of the cell depth $d$, and time is measured in units of the vertical diffusion time $d^{2} / \kappa$, where $\kappa$ is the thermal diffusivity of the fluid. The parameter $R$ is the Rayleigh number, defined to be the dimensionless temperature difference $\Delta T$ across the top and bottom plates,

$$
R=\frac{\alpha g d^{3}}{\nu \kappa} \Delta T
$$

where $\alpha$ is the thermal expansion coefficient, $\kappa$ is the thermal diffusivity, and $\nu$ is the viscous diffusivity (kinematic viscosity) of the fluid. In this paper, we will also frequently use the reduced Rayleigh number

$$
\epsilon=\frac{R-R_{c}}{R_{c}}
$$

where $R_{c} \approx 1708$ is the critical Rayleigh number at the onset of convection in an infinite domain [1]. The parameter $\sigma$ is the Prandtl number, defined to be the ratio of the fluid's thermal to viscous diffusivities,

$$
\sigma=\frac{\nu}{\kappa}
$$

The material walls are no-slip so that the velocity field satisfies

$$
\mathbf{u}=\mathbf{0} \quad \text { on all material walls. }
$$

The temperature field is constant on the top and bottom plates:

$$
T\left(x, y, z=\mp \frac{1}{2}, t\right)= \pm \frac{1}{2},
$$

and we assume that the lateral walls are perfectly insulating, so that

$$
\hat{n} \cdot \boldsymbol{\nabla} T=0 \quad \text { on lateral walls, }
$$

where $\hat{n}$ is the unit vector perpendicular to the lateral walls at a given point. The pressure field $p$ has no associated boundary condition, since it does not satisfy a dynamical equation.

The influence of the lateral walls on the dynamics is determined by the dimensionless aspect ratio $\Gamma$, defined to be the half-width-to-depth ratio of the cell if it is rectangular and the radius-to-depth ratio if it is cylindrical.

\section{B. Direct numerical simulations}

We used two different numerical schemes to solve the Boussinesq equations. The first is a serial second-orderaccurate finite-difference scheme that is based on a cubic colocated mesh. It is highly efficient for simulating a rectangular cell of moderate aspect ratio. The second is a parallel spectral element scheme that is second-order accurate in time and is able to treat more complex geometries with arbitrary lateral boundaries. Both schemes were used to obtain the results presented in this paper and were found to give good agreement with each other. Details of both of these schemes are available elsewhere [35,36]. For applications of these schemes to related problems in Rayleigh-Bénard convection, see Refs. [37-39].

\section{Mean flow}

When the convection pattern is made up of rolls that are neither concentric nor straight and parallel, a mean flow, slowly varying in the horizontal coordinates, will be set up. The importance of the mean flow is that it is a nonlocal flow mode, and as such, affects the global behavior of the convection pattern even though its magnitude is small. A detailed derivation of the mean flow can be found in Ref. [22]. Heuristically, it can be understood as follows. When there are inhomogeneities in the amplitude $A(x, y)$ and wave vector $\mathbf{k}(x, y)$ [or equivalently, the phase $\phi(x, y)$ where $\boldsymbol{\nabla}_{\perp} \phi=\mathbf{k}$ ] of the convection rolls, a Reynolds stress will be generated locally from the gradients of $\mathbf{k}$ and $A$. This results in a flow slowly varying in the plane. In addition, these inhomogeneities will also induce a varying component $p_{s}(x, y, t)$ in the pressure field that is constant across the depth of the cell and slowly varying in the plane. The gradient $\nabla_{\perp} p_{s}$ will then drive a global flow that, together with the Reynolds-stressinduced flow, distorts the convection rolls further. If we call the slowly varying flow $\mathbf{u}_{D}$, then we can write [22]

$$
\sigma \partial_{z z} \mathbf{u}_{D}=\nabla_{\perp} p_{s}+\frac{1}{2 \pi} \int_{0}^{2 \pi} d \phi \mathbf{u} \cdot \boldsymbol{\nabla} \mathbf{u}_{\perp},
$$

where the integral over the phase variable $\phi$ serves to average out the fast modes of the integrand. The as yet unknown field $p_{s}$ can be determined via the incompressibility condition, Eq. (3), which requires that

$$
\boldsymbol{\nabla}_{\perp} \cdot \int_{-1 / 2}^{1 / 2} d z \mathbf{u}_{D}(x, y, z, t)=0
$$

Equation (10) can then be integrated twice with respect to $z$, with boundary condition Eq. (7), to completely give $\mathbf{u}_{D}$. Finally, the slow distortions $\mathbf{u}_{D}$ advect the phase contours of the convection rolls, yielding an additional advection term in the phase equation [22],

$$
\partial_{t} \phi \rightarrow \partial_{t} \phi+\mathbf{U} \cdot \nabla_{\perp} \phi .
$$

The velocity field $\mathbf{U}$ is called the mean flow. It is an average of the slow distortions over the depth of the cell, 


$$
\mathbf{U}(x, y, t)=\int_{-1 / 2}^{1 / 2} d z \mathbf{u}_{D}(x, y, z, t) g(z),
$$

with $g(z)$ a weighting function that, in principle, can be calculated from the nonlinear structure of the rolls $[1,7]$.

We can approximate the mean flow from our numerical simulations as the average over the depth of the cell of the slow components of the horizontal velocity,

$$
\mathbf{U}(x, y, t) \approx \frac{1}{2 \pi} \int_{0}^{2 \pi} d \phi \int_{-1 / 2}^{1 / 2} d z \mathbf{u}_{\perp}(x, y, z, t) .
$$

In practice, we replace the integral over the phase variable $\phi$ with a Gaussian filter of characteristic width $O(1)$ so that variations over short length scales are smoothed out.

For the approximation of Eq. (14), and with the no-slip boundaries, Eq. (7), the mean flow $\mathbf{U}(x, y, t)$ is solenoidal:

$$
\nabla_{\perp} \cdot \mathbf{U}=0 .
$$

We will also find it convenient to use the mean-flow stream function $\zeta(x, y, t)$ and the vertical component of the meanflow vorticity, $\omega_{z}$, defined by

$$
-\nabla_{\perp}^{2} \zeta=\omega_{z}=\hat{z} \cdot\left(\nabla_{\perp} \times \mathbf{U}\right)
$$

The stream function, in particular, is useful to visualize because it gives the streamlines and so the geometry of the mean flow.

\section{Quenching mean flow}

We now describe a procedure to construct a modified velocity field that does not have any mean flow. To do this, we want to add to the right-hand side of Eq. (10) the negative of the source of the slow distortions, i.e., the depth average of the Reynolds stress, so that $\mathbf{u}_{D}$ becomes zero for all $(x, y, z, t)$. In Appendix A, we show that this additional term takes the form

$$
\boldsymbol{\Phi}(x, y, t)=-\rho \int_{-1 / 2}^{1 / 2} d z \mathbf{u} \cdot \nabla \mathbf{u}_{\perp}
$$

with $\rho \approx 1.5$ a constant. We can then add $\boldsymbol{\Phi}$ to the fluid equation, so that Eq. (1) becomes

$$
\sigma^{-1}\left(\partial_{t}+\mathbf{u} \cdot \boldsymbol{\nabla}\right) \mathbf{u}(x, y, z, t)=-\nabla p+\nabla^{2} \mathbf{u}+R T \hat{z}+\sigma^{-1} \boldsymbol{\Phi} .
$$

If $\boldsymbol{\Phi}$ is introduced at time $t=t_{q}$, the time needed for the modified velocity field $\mathbf{u}$ to respond to this additional forcing can be estimated by applying dimensional arguments on the terms in Eq. (18). This time scale is $O(\sigma)$. In this paper, we consider $\sigma=1$ so that we expect the mean flow to be quenched in a time scale of $O(1)$ from time $t_{q}$.

For a pattern that does not have the mean flow, such as a pattern comprising straight parallel rolls with no defects or concentric circular rolls, the quenching procedure should leave the convective properties, such as the Nusselt number, of the fluid unchanged. The Nusselt number is the ratio of convective heat transfer to heat transfer that would occur by conduction alone if the fluid remained at rest. In Fig. 1, we show that this is true. The Nusselt numbers before (denoted by solid lines) and after (dashed lines) the quenching procedure, which occurred at $t=t_{q}=50$, are indeed the same.

\section{RESULTS}

\section{A. Mean flow and spiral defect chaos}

Using the numerical schemes described in Sec. II B, we evolved Eqs. (2), (3), and (18) from the initial conditions

$$
\mathbf{u}(x, y, z, t=0)=p(x, y, z, t=0)=0
$$

and

$$
T(x, y, z, t=0)=-z+\eta(x, y, z)
$$

where $T=-z$ is the linear conduction profile and $\eta$ is randomly chosen from a uniform distribution in the range $\left[-10^{-5}, 10^{-5}\right]$. We observed spiral defect chaos when the parameters are chosen such that the reduced Rayleigh number $\epsilon$ lies in the range [0.6,3.0], the Prandtl number $\sigma \approx 1$, and the aspect ratio lies in the range [16,30]. In Fig. 2(a), we show an example: a planform of the midplane temperature field $T(x, y, z=0)$ at time $t=500$ for parameters $\epsilon=1.0, \sigma$ $=1$, and $\Gamma_{x}=\Gamma_{y}=20$. In general, the planforms we observed are qualitatively similar to those observed in experiments in both cylindrical [10] and rectangular [40] geometries.

We note that the range of aspect ratios that we have simulated is smaller than that of past experiments which extends up to $\Gamma \gtrsim 50[10,12,14]$. This limitation is caused by the need to integrate up to at least the horizontal diffusion time scale, $t \sim \Gamma^{2}$, which is an estimate of the minimum time for thermal transients to diffuse over the entire cell and thus for the pattern to reach an asymptotic state. Because of this quadratic dependence on the aspect ratio, the time needed to execute the numerical schemes so that an asymptotic pattern is reached becomes prohibitively long for $\Gamma$ beyond about 20-30 (based on current computational resources; for details, see Ref. [35]).

In the rest of this section, we report on results simulated in a rectangular cell of aspect ratio $\Gamma_{x}=\Gamma_{y}=20$. We integrated for 500 time units, and then, at time $t=t_{q}=500$, invoked the forcing term given by Eq. (17) that will quench the mean-flow dynamics. In Fig. 2(b), we plot the midplane temperature field at time $t=510$ which is ten time units after the quenching of the mean flow has begun. [Recall that the quenching takes place in a time of $O(\sigma)$ so the quenched state at ten time units should have already been asymptotic for our $\sigma=1$ state here.] We see that the rolls have "straightened out" in that they have lost their curvature and have developed angular bends. More strikingly, the straightened roll patches become stationary, leaving the only dynamics in the pattern to come from the motion of defects such as dislocations and grain boundaries. To illustrate this transition from a dynamical state to a seemingly "frozen" one, we plot in Fig. 3 the time series of the rate of change of the tempera- 


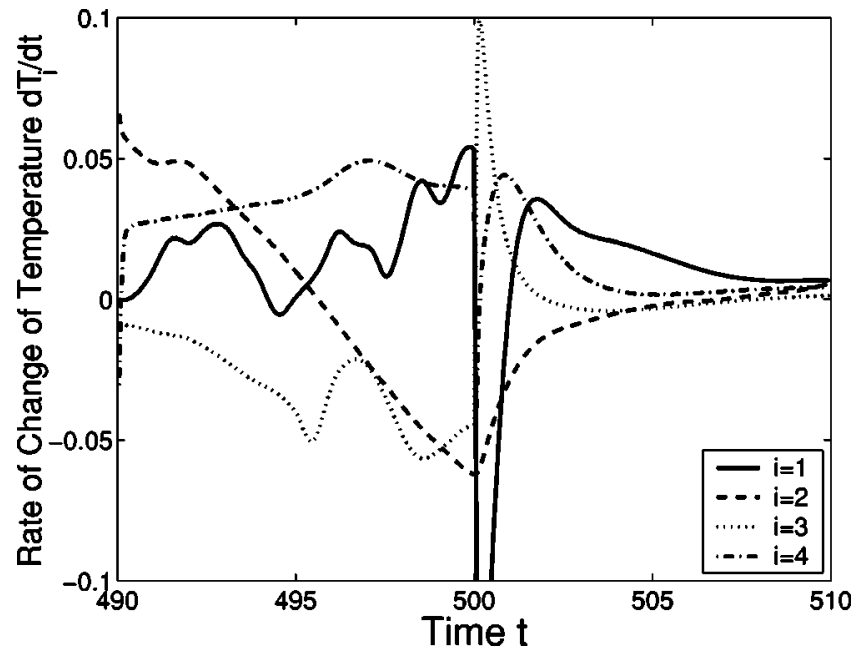

FIG. 3. The rate of change of the temperature field $d T_{i} / d t$ vs time $t$ for the four locations in the cell indicated in Fig. 2(a). Prior to quenching of the mean flow which takes place at time $t=t_{q}$ $=500$, the derivative $d T / d t$ fluctuates and differs from zero. After quenching, it approaches zero in a time scale of $O(1)$, suggesting that the pattern is approaching stationarity.

ture field at several locations in the cell. We see that, for $t$ $<t_{q}=500$, the derivative $d T(x, y) / d t$ fluctuates and is significantly different from zero at all $t<t_{q}$. However, after the quenching of the mean flow is initiated at $t=t_{q}=500$, the derivative $d T(x, y) / d t$ relaxes to approach zero in a time scale of $O(1)$, suggesting that all dynamics is becoming frozen and that a stationary pattern is being approached.

We have also repeated the quenching of the mean flow at other Rayleigh numbers ranging from $\epsilon=0.6$ to 3.0, and for different instances of the initial condition, Eq. (20). In all cases, we observed similar stationary planforms as shown in Fig. 2(b). In addition, this spiral-to-angular transition can be observed in the reverse direction. When the mean-flow quenching is turned off at a later time $t=550$ so that the mean flow is again restored to the system, the angular bends develop into spirals and the stationary planform becomes dynamical again. Spiral defect chaos is fully restored [41]. Furthermore, the stationary textures of stripes with angular bends can also be observed when the quenching is initiated at other times. For example, instead of initiating the meanflow quenching procedure at a time when a spiral defect chaotic state is already asymptotic, we have also initiated the quenching procedure immediately at the start of the simulation, $t=t_{q}=0$, again using Eqs. (19) and (20) as initial conditions. In Fig. 4(a), we show the planform after 100 time units for the parameters $\epsilon=1.0, \sigma=1$, and $\Gamma_{x}=\Gamma_{y}=20$. We see that it comprises patches of locally straight rolls ending into each other in angular bends. There are no spirals present. When the mean flow is restored at time $t=100$, we find that, after a time of $O(1)$, spiral defect chaos appears, as can be seen in Fig. 4(b) which shows the planform at 500 time units after the mean flow has been restored.

Thus, we have shown that spiral defect chaos does not exist without the presence of the mean flow. (a)

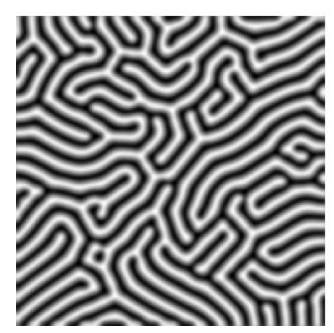

(b)

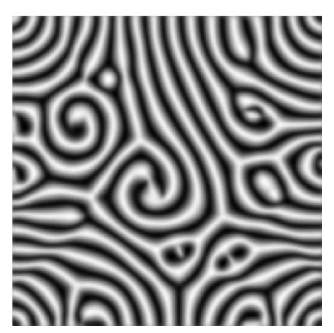

FIG. 4. (a) Stationary patches of stripes with angular bends at time $t=100$ when the mean-flow quenching is introduced at time $t=0$. The parameters are $\epsilon=1.0, \sigma=1$, and $\Gamma_{x}=\Gamma_{y}=20$. (b) When the quenching is turned off at time $t=100$ so that the mean flow is restored, spiral defect chaos is observed. The planform shown here is at 500 time units after the restoration of the mean flow.

Before we conclude this section, we qualitatively compare the differences between the states observed when the mean flow is quenched and at high Prandtl numbers, for which the mean flow is weak. (Recall that the magnitude of the mean flow is inversely proportional to the Prandtl number.) Starting from the state shown in Fig. 2(a), we instantaneously increased the Prandtl number for that state from $\sigma=1$ to $\sigma$ $=10$ at time $t=500$. Although increasing the Prandtl number changes the convective properties of the fluid and hence the dynamics of the state, we nevertheless observed [see Fig. $5(\mathrm{a})$ ] stripes with angular bends that are similar to those observed when the mean flow is quenched. Thus, the states observed when the mean flow is quenched and unquenched states observed at high Prandtl numbers are similar. In addition, we also show in Fig. 5(b) the state observed when we invoke the mean-flow quenching procedure after increasing the Prandtl number to $\sigma=10$. We see that it is again similar to the pattern at $\sigma=10$, suggesting that even at $\sigma=10$, the residual mean-flow components are negligible.

Finally, we note that, contrary to the results of Assenhe- (a)

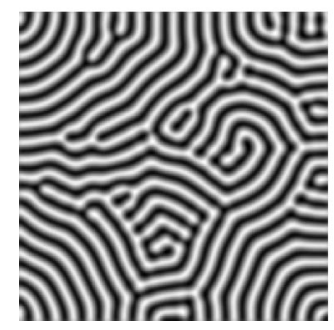

(b)

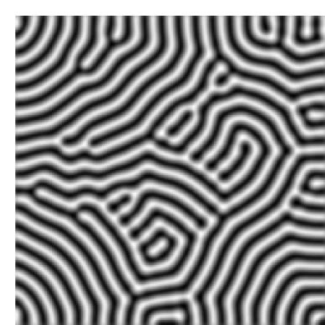

FIG. 5. (a) The pattern observed when the Prandtl number is instantaneously increased from $\sigma=1$ to $\sigma=10$ comprises stripes with angular bends that are similar to the quenched patterns in Fig. 2(a). The pattern shown here is at 100 time units after the Prandtl number has been instantaneously increased. The parameters correspond to those of the state in Fig. 2. (b) When the mean flow is quenched for the $\sigma=10$ state of (a), we see that the resulting pattern is qualitatively unchanged. Shown here is the state at 100 time units after the mean flow has been quenched. 
(a)
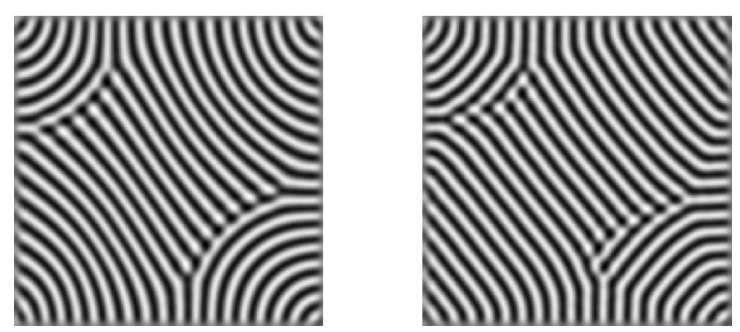

FIG. 6. (a) Midplane temperature field at time $t=500$ for parameters $\epsilon=0.15, \sigma=1$, and $\Gamma_{x}=\Gamma_{y}=20$. The stripe texture comprises patches of locally parallel rolls and arcs that are stationary. (b) The state of (a) observed at ten time units after the mean flow is quenched. The curved rolls have transitioned into stripes with angular bends that are stationary.

imer and Steinberg [20,21], we do not observe the transition from spirals to targets as the Prandtl number is increased to $\sigma=10$. Several explanations are plausible: First, in the Assenheimer and Steinberg experiments, non-Boussinesq effects are significant [20,21], whereas our direct numerical simulations are only for Boussinesq fluids. Second, our smaller aspect ratios may not support the formation of targets, and that we would indeed see the spiral to target transition in larger aspect ratios. Third, the transition to targets may be strongly dependent on the history of the system, in particular, on the path (in system space) that the parameters traverse.

\section{B. Mean flow and prechaotic stripe textures}

At lower Rayleigh numbers near the convective threshold, the planforms observed take the form of stripe textures rather than exhibiting spiral defect chaos. They comprise patches of locally parallel rolls and arcs such that each patch terminates at the boundaries of another at a different orientation, and the boundaries between the patches are usually populated by defects. In general, the stripe textures are stationary after transients, except for the motion of defects at the grain boundaries. In Fig. 6(a), we show a planform of the midplane temperature field at time $t=500$ at $\epsilon=0.15$ and $\sigma=1$ in a rectangular cell of aspect ratio $\Gamma_{x}=\Gamma_{y}=20$.

When the mean flow is quenched at time $t=t_{q}=500$, we observe that the stationary stripe textures remain stationary, and that those rolls that are curved are straightened out. The resulting pattern, shown in Fig. 6(b) which is at ten time units after the quenching, comprises patches of angular structures that replaced patches of curved arcs.

\section{Nusselt numbers}

One way to quantify the changes introduced by the quenching procedure to a pattern is to look at its global convective properties, such as the Nusselt number. For a pattern with the mean flow, the Nusselt number will be different from those of the unmodified velocity field of Eq. (18) because the latter is not a solution to the Boussinesq equations. An alternate way of saying this is that Eq. (18), together with

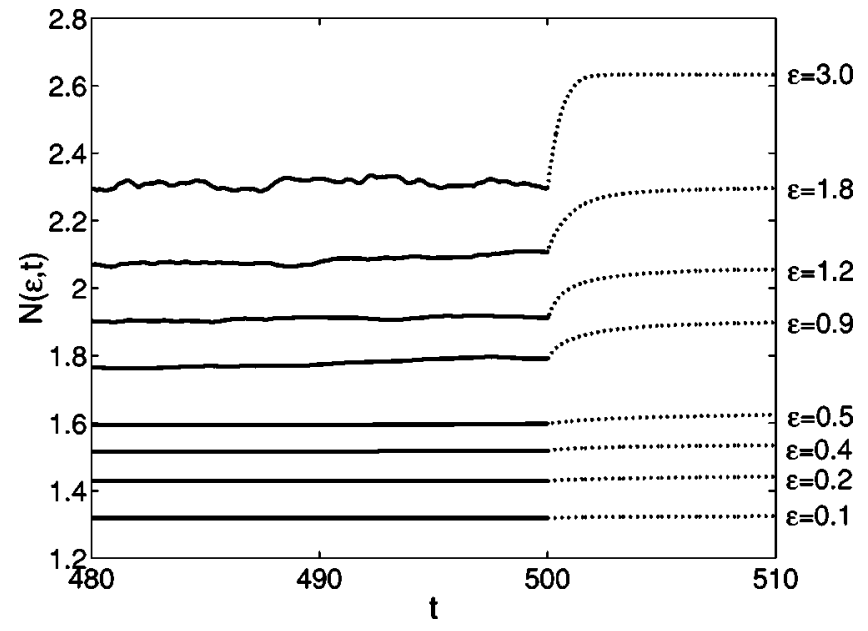

FIG. 7. Time series of the Nusselt number $N(\epsilon, t)$ for stripe textures and spiral defect chaos at several values of $\epsilon$ before (denoted by solid lines) and after (dotted lines) quenching of the mean flow which occurred at time $t_{q}=500$. All data reported here are for the Prandtl number $\sigma=1$ and a rectangular cell of aspect ratio $\Gamma_{x}$ $=\Gamma_{y}=20$.

Eqs. (2) and (3), can be interpreted as the driven Boussinesq equations with a driving force $\sigma^{-1} \boldsymbol{\Phi}$ that is turned on at time $t_{q}$. Owing to this driving, we expect the convective properties of the fluid to be stronger at time $t>t_{q}$ than at time $t$ $<t_{q}$. This is illustrated in Fig. 7. The fractional change in the Nusselt number $\Delta N / N$ caused by the introduction of the quenching of the mean flow increases with the reduced Rayleigh number. A best linear fit to the data yields the relation

$$
\Delta N / N=(0.052 \pm 0.005) \epsilon
$$

Thus, for example, when $\epsilon \sim 1$, modifying the velocity field to quench the mean flow introduces a change $\approx 5 \%$ to the averaged convective properties of the fluid.

\section{Wave number distributions}

In this section, we quantify the differences between the patterns observed with the mean flow and with the mean flow quenched by studying the wave number distributions. We compute the probability density function of wave numbers, $P(k)$, from a time average of the patterns. We used the local method discussed in Ref. [15] to calculate the wave number distributions. We have found that, for smaller aspect ratios $\Gamma \lesssim 20$, this method produces better statistics than global Fourier transform methods that were used in previous experiments $[10,12,14]$. The mean of the wave number distribution then gives the mean wave number $\langle k\rangle(\epsilon)$ as a function of the reduced Rayleigh number $\epsilon$.

Before we highlight the differences, we point out that the mean wave numbers obtained from our numerical simulations of spiral defect chaos lie within the Busse stability balloon $[1,42]$. In addition, they are also consistent with existing theory for the selection of wave numbers in spiral defect chaos $[18,19]$, which suggests that the wave numbers of convecting spirals are "frustrated," i.e., they lie between two competing selection mechanisms, selection by focus-type 


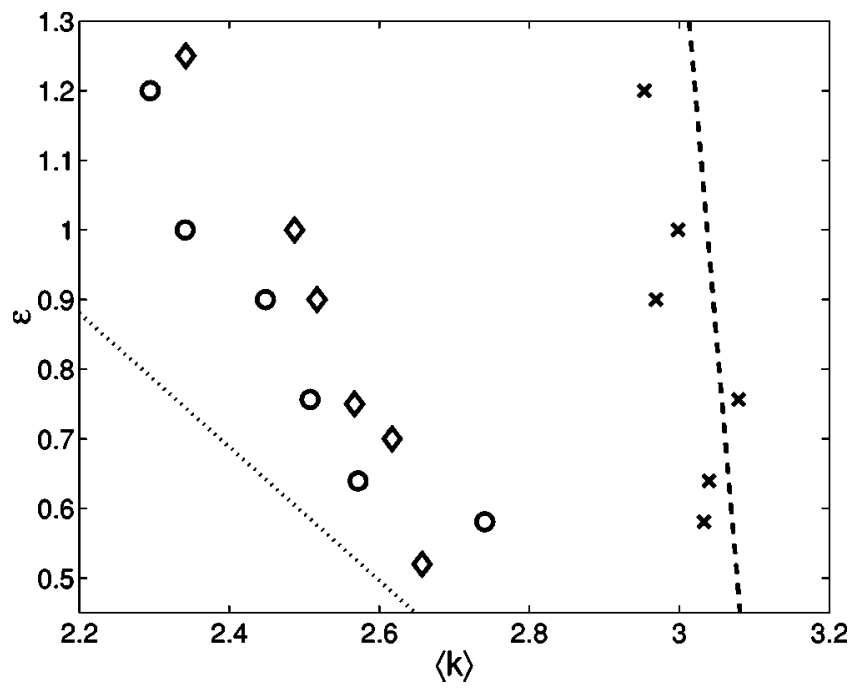

FIG. 8. Mean wave numbers $\langle k\rangle$ for various reduced Rayleigh numbers $\epsilon$. The circles denote wave numbers estimated for spiral defect chaotic states at $\sigma=1$ and $\Gamma_{x}=\Gamma_{y}=20$ from our direct numerical simulations averaged over different random initial conditions. The crosses denote wave numbers for states observed at ten time units after the mean flow is quenched. For comparison purposes, the diamonds denote wave numbers obtained in the experiment of Ref. [10] in a cylindrical cell of $\Gamma=78, \sigma=0.95$. The dashed line denotes the unique wave number $k_{f}$ possessed by focusselected convection at $\sigma=1$, and is represented by Eq. (23). The dotted line denotes the unique wave number $k_{d}$ selected by dislocations.

singularities [43] and selection by dislocations $[44,45]$. These two sets of selected wave numbers, at $\sigma=1$, are denoted in Fig. 8 by the dashed and the dotted lines, respectively. We see that our direct numerical simulations produced wave numbers (denoted by the circles) that lie within these two sets of selected wave numbers. For comparison purposes, we have also included the mean wave numbers calculated in a previous experiment $[10,14]$ performed in a cylindrical cell with $\Gamma=78$ and $\sigma=0.95$ (diamonds). We see that, at lower Rayleigh numbers, the mean wave numbers from our simulations agree with the experimental findings. However, at higher Rayleigh numbers, the wave numbers from our simulations are smaller than those of the experiments. Presumably, the smaller aspect ratios used in our simulations mean that our wave numbers are affected by finite size effects.

For the range $0.6 \leqslant \epsilon \leqslant 1.2$, the mean wave numbers of the stripes with angular bends when the mean flow is quenched (denoted by the crosses in Fig. 8) appear to fall onto a straight line whose mathematical form can be obtained from a linear fit,

$$
\langle k\rangle=(3.14 \pm 0.05)-(0.16 \pm 0.06) \epsilon .
$$

This relation is consistent with the wave numbers selected by focus-type singularities at Prandtl number $\sigma=1$ [43],

$$
k_{f}=3.117-0.13 \epsilon
$$

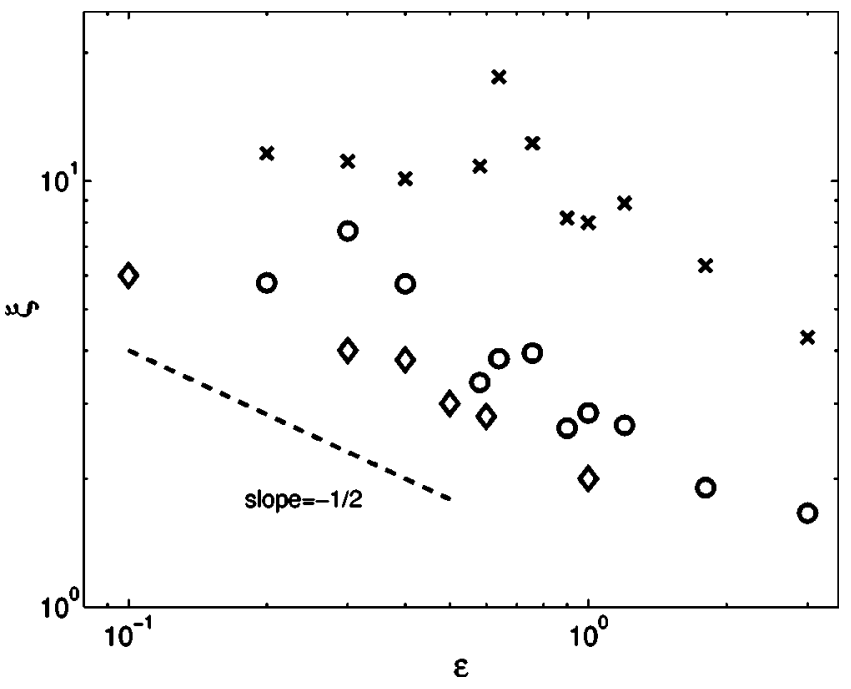

FIG. 9. Correlation length $\xi$ vs the reduced Rayleigh number $\epsilon$. The symbols are as defined in Fig. 8. The dashed line corresponds to the power law $\xi \propto \epsilon^{-1 / 2}$.

The local pattern in focus-selected convection includes rolls that form closed contours about a point within the cell. In our rectangular geometries, the four corners act as focus centers, as can be seen by the presence of approximately axisymmetric roll patches emanating from the corners, see Fig. 2(a). In the absence of the mean flow, the wave number selected therefore appears to be dominated by that selected by the focus centers (i.e., the corners) to give a mean wave number consistent with that selected in focus-type singularities.

Furthermore, in the absence of the mean flow, the wave numbers $k_{f}$ lie at the boundary of the zigzag instability [22]. The patterns observed with the mean flow quenched are thus dominated by lateral "zig and zag" bendings, leading to the stripes with angular bends observed in Figs. 2(b) and 6(b).

We have also computed the correlation length $\xi(\epsilon)$ of the patterns as a function of $\epsilon$. The correlation length, defined here as the inverse of the standard deviation of the probability density function $P(k)$, is a measure of the average length scale of correlated regions in the pattern. In Fig. 9, we show $\xi(\epsilon)$ calculated for both unquenched patterns (denoted by the circles in Fig. 9) and for patterns observed when the mean flow is quenched (crosses). For comparison purposes, we have also included the correlation lengths calculated from a previous experiment $[10,14]$ performed in a cylindrical cell with $\Gamma=78$ and $\sigma=0.95$ (diamonds). We see that the correlation lengths for the states when the mean flow is quenched are, on the average, about twice as large as those for spiral defect chaos at all values of $\epsilon$. In addition, the correlation lengths for the unquenched patterns can be fitted with the power law $\xi \propto \epsilon^{-1 / 2}$, as has been suggested by past experiments $[12,14]$, and which is predicted by dimensional arguments to be valid at least near threshold. However, the same cannot be said for the quenched states. In fact, the data suggest that while an exponent of $-1 / 2$ might be fitted for $\epsilon$ $\gtrsim 0.7$, the correlation lengths appear to have saturated at $\xi$ $\sim \Gamma=14$ for $\epsilon \lessgtr 0.7$. This suggests that finite size effects become important, and that, in order to obtain a better estimate of the scaling relation for the patterns observed when the 


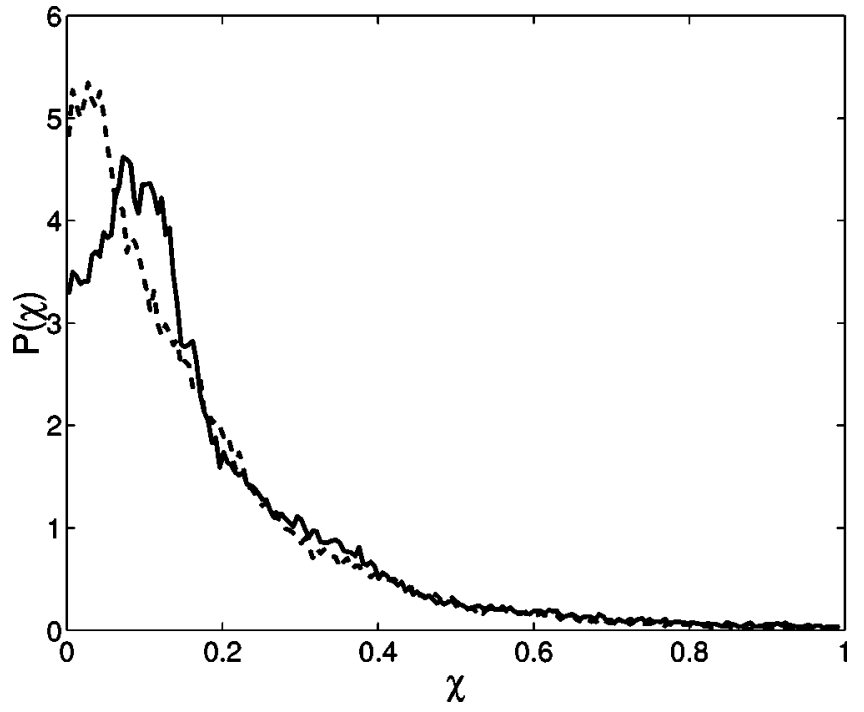

FIG. 10. The probability density function $P(\chi)$ of the curvature $\chi$. The solid line is for the spiral defect chaotic state at $\epsilon=1.0$, $\sigma=1$, and $\Gamma_{x}=\Gamma_{y}=20$ averaged over different random initial conditions and times $t=400-500$. The dashed line is for the stripes with angular bends observed at ten time units after the mean flow has been quenched.

mean flow is quenched, we would need to use a larger aspect ratio. Owing to the lack of data over more decades of the reduced Rayleigh numbers, actual fittings to the data were not carried out.

\section{E. Curvature distributions}

Finally, we quantify how much the quenching of the mean flow straightens the rolls by looking at the distribution of the local curvature $\chi$, defined at every point in the planform to be the magnitude of the divergence of the unit wave vector:

$$
\chi=|\nabla \cdot \hat{k}|
$$

A value of $\chi=0$ corresponds to a straight roll, whereas a value of $\chi=1$ corresponds to a roll with a radius of curvature of unity.

We have computed the probability density function $P(\chi)$ for spiral defect chaos observed at $\epsilon=1.0, \sigma=1$, and $\Gamma_{x}$ $=\Gamma_{y}=20$, as well as for the resulting stripes with angular bends observed at ten time units after the mean flow is quenched. In Fig. 10, we plot the two distributions. The curvature distribution for spiral defect chaos (solid line) peaks at a value of $\chi \approx 0.1$, suggesting that the pattern is dominated by spirals whose radius of curvature is $\chi^{-1} \sim 10$, consistent visually with the pattern shown in Fig. 2(a). We see that this peak broadens to become a plateau at $0 \leqq \chi \lesssim 0.1$ for the quenched state (dashed line), suggesting an increase in the dominance of straighter rolls in the pattern.

We observed similar results for the comparison of the curvature distribution for the stripe textures. In Fig. 11, we show the comparisons for a state at $\epsilon=0.15$. We see that both distributions, with (solid line) and without (dashed line) the mean flow, decrease approximately monotonically and

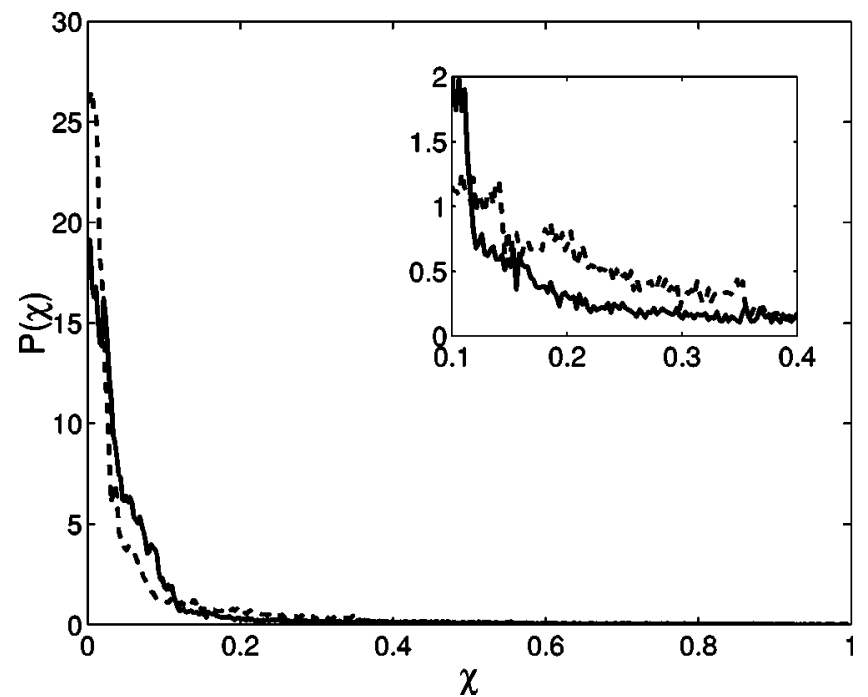

FIG. 11. The probability density function $P(\chi)$ of the curvature $\chi$. The solid line is for stripe textures at $\epsilon=0.15, \sigma=1$, and $\Gamma_{x}$ $=\Gamma_{y}=20$ averaged over different random initial conditions and times $t=400-500$. The dashed line is for the stripes with angular bends observed at 10 times units after the mean flow has been quenched. Because $P(\chi) \approx 0$ for $\chi \gtrsim 0.5$, the region $0.5 \lesssim \chi \lesssim 1$ is not plotted. The inset shows the region $0.1 \leqslant \chi \leqslant 0.4$ enlarged.

rapidly with increasing $\chi$. Both the comparisons for spiral defect chaos and for stripe textures suggest that the consequence of quenching the mean flow is to straighten out the rolls.

In addition, the distribution at $\epsilon=0.15$ for the quenched case is higher for $\chi \lesssim 0.05$ as well as for $0.1 \lessgtr \chi \lesssim 0.4$ (see the inset of Fig. 11), and lower otherwise. This suggests that another consequence of quenching the mean flow is the development of angular structures that have large curvatures.

\section{F. Mean flow and lateral boundaries}

In experiments where the Rayleigh number is sufficiently high, it has been frequently observed that convection rolls terminate perpendicularly into the lateral walls. We show in this section that the mean flow generated by amplitude gradients near lateral walls can be used to explain this phenomenon, although the applicability of this argument rests on a number of factors, among them the presence of defects which affects the ability of the patterns to reorient themselves.

If we call $\hat{n}$ the outward unit vector normal to the lateral boundary and $\hat{k}$ the wave director of the rolls, then we can define the wall-roll obliqueness angle as

$$
\Theta \equiv \arccos |\hat{k} \cdot \hat{n}|
$$

In practice, the numerical value of $\Theta$ at a particular location along the lateral boundary is obtained by averaging Eq. (25) over a length $r=0.5$ to $r=1.5$, where $r$ is the perpendicular distance away from that location along the lateral boundary. The value $\Theta=\pi / 2$ corresponds to rolls terminating perpendicularly into the walls. The common occurrence of this 


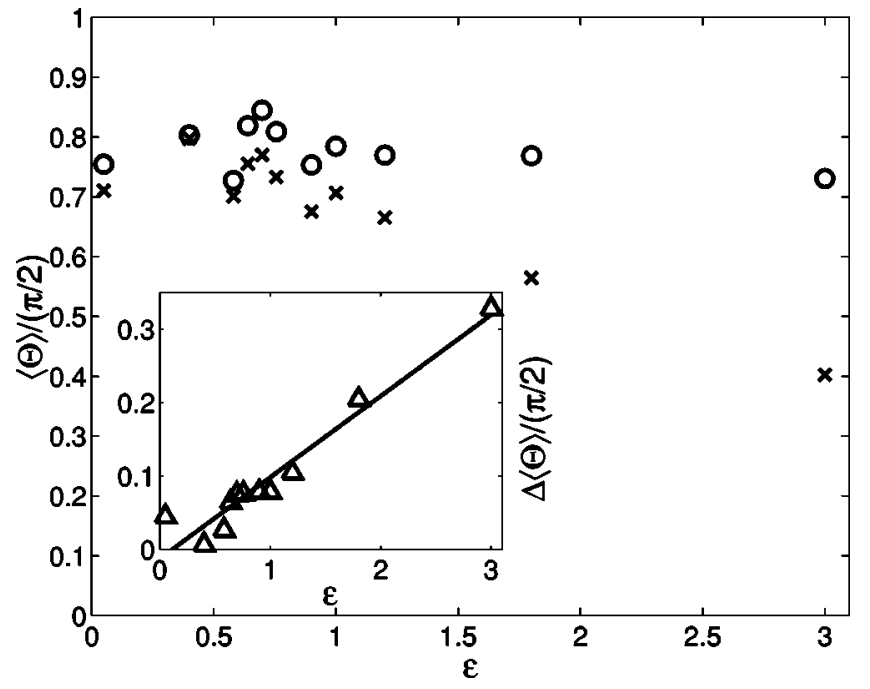

FIG. 12. The mean wall-roll obliqueness angle $\langle\Theta\rangle$ as a function of the reduced Rayleigh number $\epsilon$ for states with the mean flow (circles) and with the mean flow quenched (crosses). The inset shows the difference between the two sets of data, $\Delta\langle\Theta\rangle$, as a function of $\epsilon$.

value remains a phenomenological observation, without much theoretical understanding, although it has been found [46] that $\Theta$ is not fixed by the physical boundary conditions, Eqs. (7)-(9).

However, when rolls do not terminate perpendicularly at a lateral boundary, variations in the amplitude of the convection rolls as it decays near the lateral boundaries result in the generation of a mean flow. [Recall from Eq. (10) that a mean flow is generated by inhomogeneities in the wave numbers and amplitudes of the convection rolls.] The normal component (with respect to the lateral boundary) of this mean flow is canceled by the flow generated from slow pressure gradients, resulting in the mean flow being parallel to the lateral boundary. It then tends to push the rolls back to a perpendicular orientation. The actual calculations are worked out in Appendix B. The importance of this mean flow in ensuring that the rolls terminate perpendicularly is indicated by observing that, in the absence of the mean flow, oblique rolls are more prevalent. In Fig. 12, we plot the wall-roll obliqueness angle averaged over the lateral boundaries for patterns observed at $t=500$ at various reduced Rayleigh number $\epsilon$, Prandtl number $\sigma=1$, and in a rectangular cell of aspect ratio $\Gamma_{x}=\Gamma_{y}=20$, with the mean flow and with the mean flow quenched. We see that, with the mean flow, the rolls are close to perpendicular, $\Theta \approx \pi / 2$. However, when the mean flow is quenched, the rolls are more oblique, $\Theta \leqq \pi / 2$. In fact, the difference in the mean wall-roll obliqueness angle between the states with the mean flow and with the mean flow quenched, $\Delta\langle\Theta\rangle$, increases approximately linearly with $\epsilon$,

$$
\Delta\langle\Theta\rangle=(0.16 \pm 0.01) \epsilon,
$$

as the inset of Fig. 12 depicts.

When the mean flow is quenched, the reorientation of the rolls away from $\Theta=\pi / 2$ is almost instantaneous. We illus-

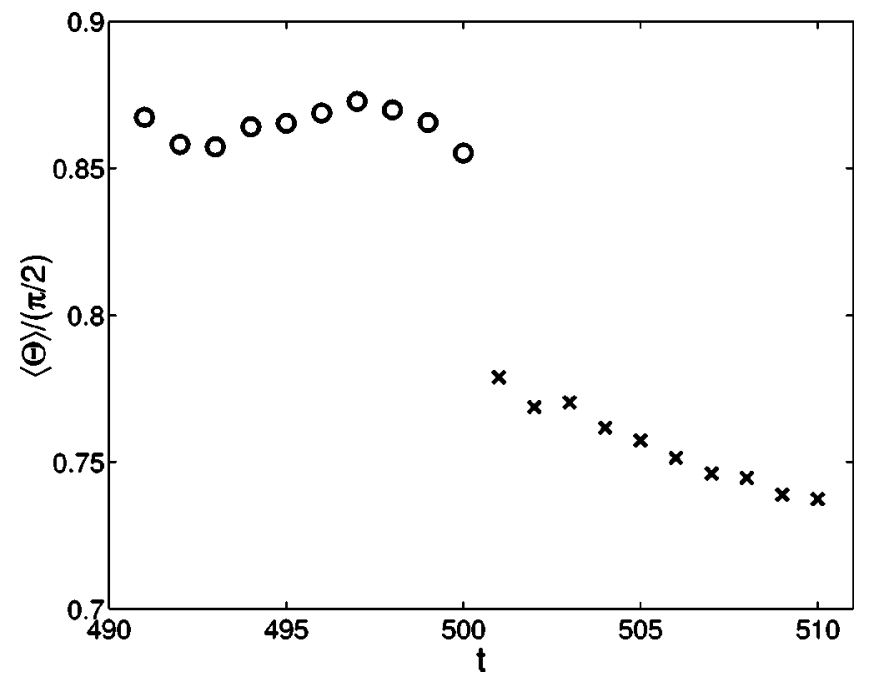

FIG. 13. The change in the mean wall-roll obliqueness angle $\langle\Theta\rangle$ as a function of time, averaged over different random initial conditions. The parameters here are $\epsilon=1.0, \sigma=1$, and $\Gamma_{x}=\Gamma_{y}$ $=20$. The mean-flow quenching takes place at time $t=t_{q}=500$, so that, for $490 \leqslant t \leqslant 500$, the mean wall-roll obliqueness angle is for a pattern whose bulk dynamics exhibits spiral defect chaos, whereas for $500 \leqslant t \leqslant 510$, the bulk dynamics is made up of stripes with angular bends.

trate this result in Fig. 13 for one particular reduced Rayleigh number $\epsilon=1.0$. In this case, the mean-flow quenching takes place at time $t=t_{q}=500$. We see that, at time $t=500$, the mean wall-roll obliqueness angle moves away from $\Theta$ $=\pi / 2$ in a time scale of $O(1)$.

The above argument that the mean flow restores the rolls to a perpendicular orientation may not always be applicable. For example, when we performed simulations in a cylindrical cell of aspect ratio $\Gamma=30$, we find that, at $\epsilon=1.0$, the mean wall-roll obliqueness angle $\langle\Theta\rangle$ still remains close to $\pi / 2$ when the mean flow is quenched. This can be seen more clearly in Fig. 14, where we show the probability density $P(\Theta)$ of wall-roll obliqueness angles along the lateral boundaries for states observed in a rectangular cell of aspect ratio $\Gamma_{x}=\Gamma_{y}=20$ and cylindrical cell of aspect ratio $\Gamma$ $=30$. We see that in a cylindrical cell with the mean flow quenched, the peak at $\Theta \approx \pi / 2$ is still observed after the mean flow has been quenched. One possible explanation might be that, in a cylindrical cell, there are more defects existing near the lateral boundaries and that these defects then pin the rolls, preventing them from reorienting away from $\Theta=\pi / 2$ when the mean flow is quenched.

Another instance where the above argument does not apply is at low Rayleigh numbers. From Eq. (B7) in Appendix $\mathrm{B}$, the magnitude of the mean flow $|\mathbf{U}| \propto \epsilon^{1 / 2}$ so that at low Rayleigh numbers, the mean flow may not be strong enough to reorient the rolls perpendicularly. This is evident in Fig. 6(a), where, at the reduced Rayleigh number $\epsilon=0.15$, rolls are seen to terminate with an acute angle at the lateral walls. In this case, the presence of a restoring mean flow can also be visualized. The mean-flow vorticity plot corresponding to this pattern, shown in Fig. 15(a), shows the presence of strong vorticity along the bottom half of the left wall and the 
(a)

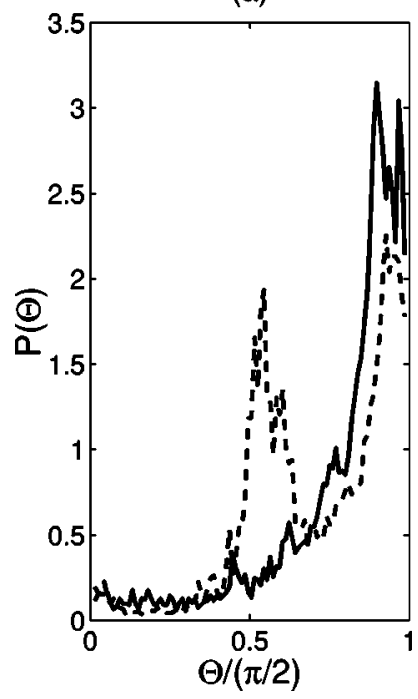

(b)

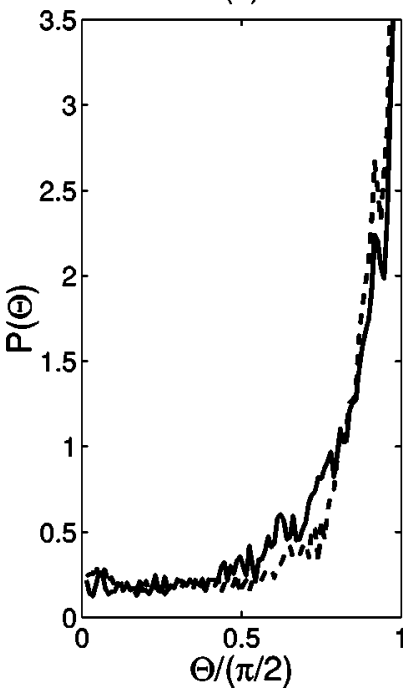

FIG. 14. (a) Distribution of angles where rolls terminate at a lateral boundary in a rectangular cell of aspect ratio $\Gamma_{x}=\Gamma_{y}=20$. The solid line shows the distribution for the spiral defect chaotic state averaged over different initial conditions at $\epsilon=1.0$ and $\sigma$ $=1$. The dashed line shows the distribution for the state with the mean flow quenched. (b) The solid line shows the distribution for spiral defect chaos observed in a cylindrical cell of aspect ratio $\Gamma$ $=30$ at $\epsilon=1.0$ and $\sigma=1$. The dashed line shows the distribution for this state but with the mean flow quenched.

top half of the right wall. There, the restoring mean-flow vorticity takes the form of long and narrow circulating "jets" that are about one roll size wide and several roll sizes long. In Fig. 15(b), the vorticity is plotted as a function of distance away from the lateral wall along the solid and dashed horizontal lines shown in Fig. 15(a). The existence of a positive vorticity patch close to the wall and a negative patch further away from the wall, which together indicate the presence of a restoring mean flow, agrees qualitatively with the theoretical results of Fig. 18 in Appendix B. When the Rayleigh

(a)

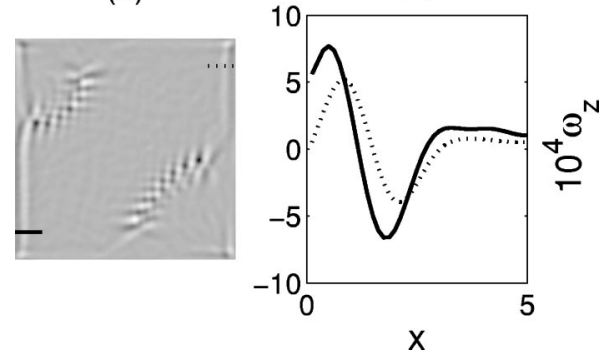

FIG. 15. (a) The mean-flow vorticity $\omega_{z}(x, y)$ corresponding to the stripe texture of Fig. 6(a) obtained using Eq. (16). Light regions correspond to positive vorticity, dark regions to negative vorticity. The important feature in this vorticity map is the presence of "jet"like structures along the bottom half of the left wall and the top half of the right wall. (b) The vorticity $\omega_{z}(x)$ is plotted along the solid and the dashed horizontal lines shown in (a). The shape of $\omega_{z}(x)$ is to be compared with Fig. 18 in Appendix B, where a positive and a negative vorticity patch sets up a restoring mean flow.

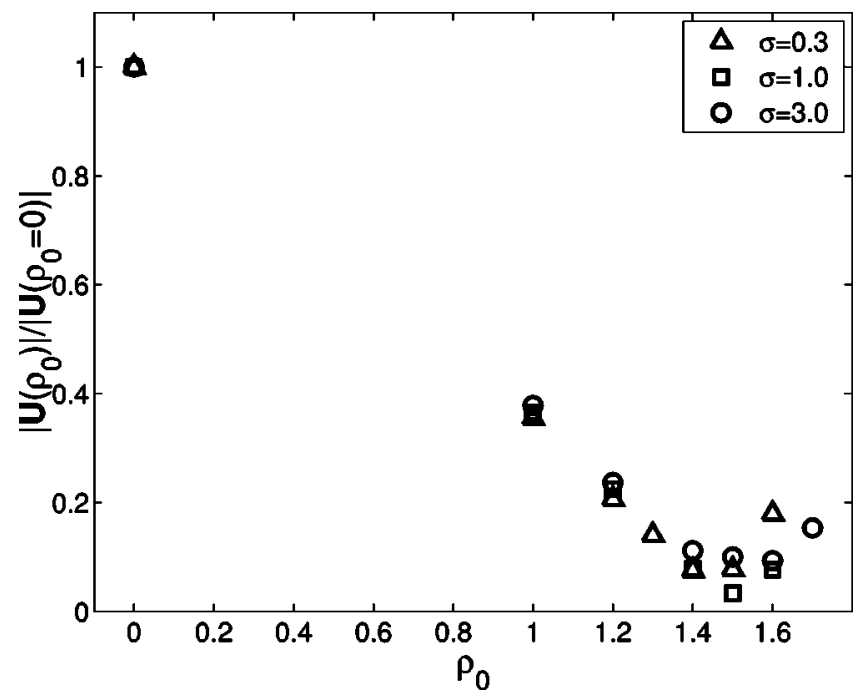

FIG. 16. The maximum mean-flow magnitude vs various trial values of $\rho$. The mean-flow magnitudes are normalized by their values at $\rho=0$, i.e., when there is no quenching. We see that, when $\rho \approx 1.5$, the mean flow goes to zero, for all three Prandtl numbers.

number of the state in Fig. 6 is increased from $\epsilon=0.15$ to $\epsilon=1.0$, the mean flow becomes strong enough to reorient the rolls to become perpendicular to the lateral walls, and subsequently disappears.

\section{CONCLUSION}

In this paper, we have described a procedure to construct a modified velocity field that does not have any mean flow in a convecting flow. We have applied this procedure to show that spiral defect chaos does not survive when the mean flow is quenched. Instead, a pattern characterized by textures of stripes with angular bends appears. We have also shown that the mean wave numbers of these quenched patterns approach those selected by focus-type singularities, which, in the absence of the mean flow, lie at the boundary of the zigzag instability.

We next presented a heuristic argument on how the mean flow can contribute to rolls terminating into a lateral boundary perpendicularly. We provided data to show that, in the absence of the mean flow, the rolls begin to deviate from a perpendicular orientation, and this obliqueness increases with the Rayleigh number. However, the ability of this mean flow to restore the rolls to a perpendicular orientation may be impeded by the presence of defects that do not allow the rolls to reorient themselves, and at low Rayleigh numbers where the restoring mean flow is weak.

\section{ACKNOWLEDGMENTS}

This work was supported by the Engineering Research Program of the Office of Basic Energy Sciences at the U.S. Department of Energy, Grant Nos. DE-FG03-98ER14891 and DE-FG02-98ER14892. We acknowledge the Caltech Center for Advanced Computing Research and the North Carolina Supercomputing Center. We also thank Paul Fischer for helpful discussions. 


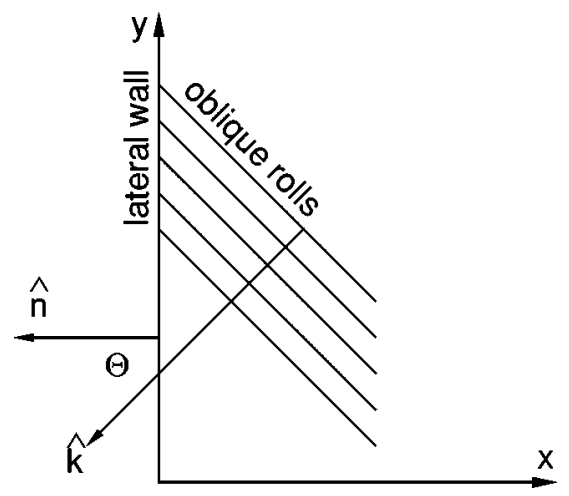

FIG. 17. Straight and parallel convection rolls with wave director $\hat{k}$ terminating at a lateral boundary with outward normal $\hat{n}$ at an angle of obliqueness $\Theta$. Note that, by our definition, $0<\Theta<\pi / 2$. The perpendicular distance away from the lateral wall is $x$.

\section{APPENDIX A: DERIVATION OF QUENCHING OF THE MEAN FLOW}

In this appendix, we derive the functional form of the forcing term $\boldsymbol{\Phi}$ that is to be added to the fluid equation, Eq. (1), to make the resulting fluid dynamics have zero mean flow.

As mentioned in Sec. II C, the mean flow comprises a local component generated by the Reynolds stress $1 /(2 \pi) \int_{0}^{2 \pi} d \phi \mathbf{u} \cdot \nabla_{\perp} \mathbf{u}_{\perp}$ and a global component driven by a slow horizontal pressure gradient that is present in order to guarantee the incompressibility condition, Eq. (3). Thus, if the Reynolds stress is subtracted from the dynamics at all times, then the mean flow will not be generated. We thus suggest that

$$
\boldsymbol{\Phi}(x, y, t)=\frac{1}{2 \pi} \int_{0}^{2 \pi} d \phi \rho \int_{-1 / 2}^{1 / 2} d z \mathbf{u} \cdot \nabla_{\perp} \mathbf{u}_{\perp},
$$

where the operator $\rho \int_{-1 / 2}^{1 / 2} d z$ serves as an average over the depth of the cell. This $\boldsymbol{\Phi}$ can then be subtracted from the fluid equation, Eq. (1), resulting in Eq. (18).

We now need to evaluate the value of the constant $\rho$. To do this, we rewrite the equation for the slow distortions, Eq. (10), as

$$
\sigma \partial_{z z} \mathbf{u}_{D}=\nabla_{\perp} p_{s}+\frac{1}{2 \pi} \int_{0}^{2 \pi} d \phi \mathbf{u} \cdot \boldsymbol{\nabla} \mathbf{u}_{\perp}-\boldsymbol{\Phi} .
$$

Following Ref. [22], the Reynolds stress term near threshold takes the form

$$
\frac{1}{2 \pi} \int_{0}^{2 \pi} d \phi \mathbf{u} \cdot \nabla \mathbf{u}_{\perp} \equiv I(k, z) \mathbf{R}(x, y),
$$

where

$$
\mathbf{R}(x, y) \equiv \mathbf{k} \boldsymbol{\nabla}_{\perp} \cdot\left(\mathbf{k} A^{2}\right),
$$

and
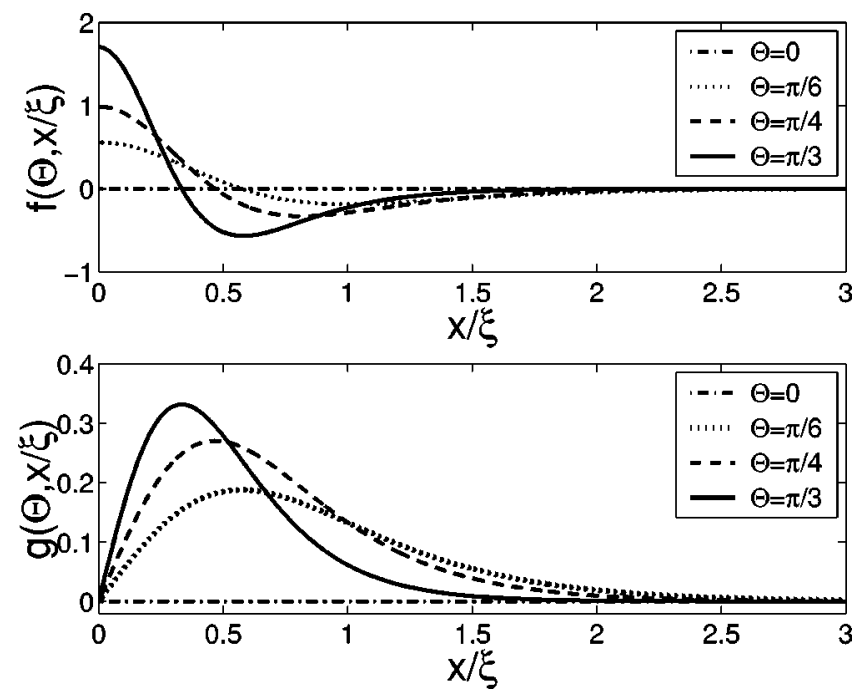

FIG. 18. (a) The function $f(\Theta, x)$ defined in Eq. (B5) which is the normalized vertical component of the mean-flow vorticity. It is plotted here for several values of $\Theta$ and vs $x / \xi$. (b) The function $f(\Theta, x)$ defined in Eq. (B8) which is the normalized mean-flow magnitude. It is plotted here for several values of $\Theta$ and vs $x / \xi$.

$$
I(k, z) \equiv w_{0}(k, z) \partial_{z} \frac{\partial \phi_{0}(k, z)}{\partial k_{c}^{2}}-\frac{\partial w_{0}(k, z)}{\partial k_{c}^{2}} \partial_{z} \phi_{0}(k, z),
$$

with $w_{0}(k, z)$ and $\phi(k, z)$ the vertical profiles of the vertical velocity and the potential of the horizontal velocities, respectively. For systems satisfying the rigid boundary condition, Eq. (7), these functions are the familiar Chandrasekhar functions [47].

We can then rewrite Eq. (A2) as

$$
\sigma \partial_{z z} \mathbf{u}_{D}=\nabla_{\perp} p_{s}+I(k, z) \mathbf{R}(x, y)-\rho \int_{-1 / 2}^{1 / 2} d z I(k, z) \mathbf{R}(x, y)
$$

Integrating Eq. (A6) with respect to $z$ twice, and making use of the boundary condition, Eq. (7),

$$
\begin{aligned}
\sigma \mathbf{u}_{D}= & p(z) \boldsymbol{\nabla}_{\perp} p_{s}+J(k, z) \mathbf{R}(x, y) \\
& -p(z) \rho \int_{-1 / 2}^{1 / 2} d z I(k, z) \mathbf{R}(x, y),
\end{aligned}
$$

with

$$
p(z) \equiv \frac{1}{2}\left(z^{2}-\frac{1}{4}\right)
$$

the Poiseuille profile, and $J(k, z)$ the double integral of $I(k, z)$ with respect to $z$. Employing the incompressibility condition, Eq. (11), we then arrive at the equality 


$$
\rho=\frac{12 \int_{-1 / 2}^{1 / 2} d z J(k, z)}{\int_{-1 / 2}^{1 / 2} d z I(k, z)} .
$$

Evaluating these integrals numerically yields $\rho \approx 1.5$ for the rigid boundary condition, Eq. (7). Moreover, $\rho$ is relatively independent of the wave number $k$, varying from $\rho$ $=1.4886$ at $k=2.8$ to $\rho=1.4887$ at $k=k_{c}=3.117$ and to $\rho$ $=1.4886$ at $k=3.4$, suggesting the validity of treating it as a constant.

Finally, to numerically confirm this result, we carry out the quenching of the mean flow, as described in Eq. (18), for a range of values for $\rho$, at $\epsilon=1.0$ in a rectangular cell of $\Gamma_{x}=\Gamma_{y}=20$. At ten time units after affecting the quenching, we then measure the maximum magnitude of the mean flow as a function of $\rho$. We plot our results in Fig. 16, where we show the maximum mean-flow magnitude (normalized by the maximum mean-flow magnitude observed without quenching) vs $\rho$ for data from three different Prandtl numbers. We see that, when $\rho \approx 1.5$, the normalized maximum mean-flow magnitude is indeed zero.

\section{APPENDIX B: RESTORING THE MEAN FLOW NEAR A LATERAL BOUNDARY}

In this appendix, we show that a set of straight and parallel rolls that are oriented obliquely at an angle $\Theta$ to a lateral boundary sets up a mean flow that tends to restore the rolls back to being perpendicular to the lateral boundary. The various quantities used here are defined in the sketch in Fig. 17.

We will make the assumption that the wave vectors of the rolls are constant near the lateral boundary,

$$
\mathbf{k}=(-k \cos \Theta,-k \sin \Theta)
$$

and that the convection amplitude within a correlation length $\xi$ of a lateral boundary is suppressed $[1,48]$ :

$$
A(x, y)=A_{0} \tanh \left(\frac{x}{\xi \cos \Theta}\right) \text {. }
$$

The quantity $A_{0}$ is the amplitude in the bulk. The correlation length $\xi=\sqrt{2} \epsilon^{-1 / 2} \xi_{0}$ with $\xi_{0}=0.385$. The variable $x$ is the perpendicular distance away from the lateral boundary.

Then, from the Cross-Newell equation [22], the amplitude gradients near the lateral wall will result in a non zero meanflow vorticity $\omega$ given by

$$
\omega=\gamma \hat{z} \cdot \nabla_{\perp} \times\left[\mathbf{k} \nabla_{\perp} \cdot\left(\mathbf{k} A^{2}\right)\right]
$$

where $\gamma$ is a constant that is inversely proportional to the Prandtl number $\sigma$. (If we relax the assumption that the wave numbers of the rolls are constant, then the compression and dilation of the rolls as well as inhomogeneities in their curvatures will also contribute to the mean flow.) Substituting Eqs. (B1) and (B2) into Eq. (B3) then gives

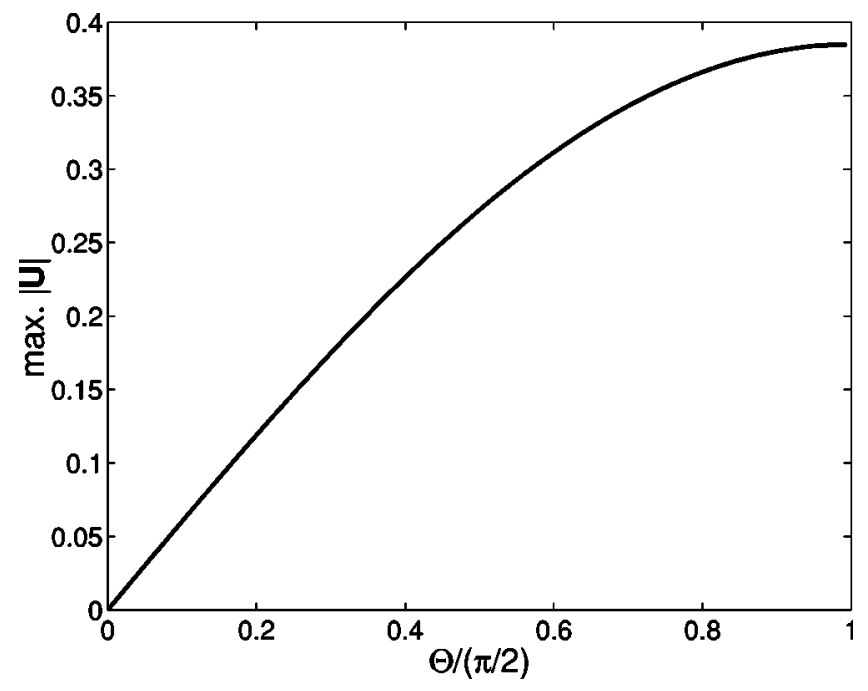

FIG. 19. The maximum magnitude of the mean flow $\mathbf{U}$ as a function of the wall-roll obliqueness angle $\Theta$. It increases monotonically from zero at $\Theta=0$ (rolls parallel to the wall).

$$
\omega(x)=2 \gamma A_{0}^{2} k^{2} \xi^{-2} f(\Theta, x),
$$

where the normalized mean-flow vorticity

$$
f(\Theta, x)=\tan (\Theta) \operatorname{sech}^{2}\left(\frac{x}{\xi \cos \Theta}\right)\left[1-3 \tanh ^{2}\left(\frac{x}{\xi \cos \Theta}\right)\right],
$$

is plotted in Fig. 18(a) for several representative values of $\Theta$. We see that $\omega$ is positive for $x / \xi \lesssim 1$, and negative otherwise. The currents from this vorticity pair will then drive the rolls back to a perpendicular orientation.

The mean flow generated by this vorticity can also be easily computed. Along the lateral wall, it is given by

$$
|\mathbf{U}|=\gamma\left|\mathbf{k}_{y}\right| \boldsymbol{\nabla}_{\perp} \cdot\left(\mathbf{k} A^{2}\right) .
$$

(The component of the mean flow normal to the lateral wall is canceled by the flow coming from the slow pressure gradient.) Using Eqs. (B1) and (B2), we arrive at

$$
|\mathbf{U}|=2 \gamma A_{0}^{2} k^{2} \xi^{-1} g(\Theta, x),
$$

where the normalized restoring mean-flow magnitude in the direction of the lateral wall,

$$
g(\Theta, x)=\sin (\Theta) \operatorname{sech}^{2}\left(\frac{x}{\xi \cos \Theta}\right) \tanh \left(\frac{x}{\xi \cos \Theta}\right),
$$

is plotted in Fig. 18(b) for several representative values of $\Theta$.

Finally, we plot the quantity $\max |\mathbf{U}|$ as a function of $\Theta$ in Fig. 19. We see that the restoring mean-flow magnitude grows monotonically from zero at $\Theta=0$ (corresponding to sets of rolls parallel to the lateral wall) to attain its largest value at $\Theta \rightarrow \pi / 2$ (corresponding to sets of rolls perpendicular to the wall). Our analysis actually breaks down for $\mid \Theta$ $-\pi / 2 \mid \lesssim \epsilon^{1 / 4}$ because modifications at the next order in Eq. (B2) become important [46]. 
[1] M.C. Cross and P.C. Hohenberg, Rev. Mod. Phys. 65, 851 (1993).

[2] M.C. Cross and P.C. Hohenberg, Science (Washington, DC, U.S.) 263, 1569 (1994).

[3] J.P. Gollub and M.C. Cross, Nature (London) 404, 710 (2000).

[4] P.C. Hohenberg and B.I. Shraiman, Physica D 37, 109 (1989).

[5] E.D. Siggia and A. Zippelius, Phys. Rev. Lett. 47, 835 (1981).

[6] M.C. Cross, Phys. Rev. A 27, 490 (1983).

[7] A.C. Newell, T. Passot and M. Souli, J. Fluid Mech. 220, 187 (1990)

[8] A.C. Newell, T. Passot, and M. Souli, Phys. Rev. Lett. 64, 2378 (1990).

[9] A.C. Newell, T. Passot, and J. Lega, Annu. Rev. Fluid Mech. 25, 339 (1993).

[10] S.W. Morris, E. Bodenschatz, D.S. Cannell, and G. Ahlers, Phys. Rev. Lett. 71, 2026 (1993).

[11] Y. Hu, R. Ecke, and G. Ahlers, Phys. Rev. Lett. 74, 391 (1995).

[12] Y. Hu, R. Ecke, and G. Ahlers, Phys. Rev. E 51, 3263 (1995).

[13] J. Liu and G. Ahlers, Phys. Rev. Lett. 77, 3126 (1996); 77, 4853 (1996).

[14] S.W. Morris, E. Bodenschatz, D.S. Cannell and G. Ahlers, Physica D 97, 164 (1996).

[15] D.A. Egolf, I.V. Melnikov, and E. Bodenschatz, Phys. Rev. Lett. 80, 3228 (1998).

[16] R.E. Ecke, Y. Hu, R. Mainieri, and G. Ahlers, Science (Washington, DC, U.S.) 269, 1704 (1995).

[17] D.A. Egolf, I.V. Melnikov, W. Pesch, and R.E. Ecke, Nature (London) 404, 733 (2000).

[18] M.C. Cross and Y. Tu, Phys. Rev. Lett. 75, 834 (1995).

[19] M.C. Cross, Physica D 97, 65 (1996).

[20] M. Assenheimer and V. Steinberg, Phys. Rev. Lett. 70, 3888 (1993).

[21] M. Assenheimer and V. Steinberg, Nature (London) 367, 345 (1994)

[22] M.C. Cross and A.C. Newell, Physica D 10, 299 (1984).

[23] J. Swift and P.C. Hohenberg, Phys. Rev. A 15, 319 (1977).

[24] H.S. Greenside, M.C. Cross, and W.M. Coughran, Jr., Phys. Rev. Lett. 60, 2269 (1988).
[25] H. Xi, J.D. Gunton, and J. Viñals, Phys. Rev. Lett. 71, 2030 (1993).

[26] H. Xi and J.D. Gunton, Phys. Rev. E 52, 4963 (1995).

[27] X. Li, H. Xi, and J.D. Gunton, Phys. Rev. E 57, 1705 (1998).

[28] L.S. Tsimring, Physica A 249, 125 (1998).

[29] R. Schmitz, W. Zimmermann, and W. Pesch, Phys. Rev. E 65, 037302 (2002).

[30] W. Decker, W. Pesch, and A. Weber, Phys. Rev. Lett. 73, 648 (1994).

[31] W. Pesch, Chaos 6, 348 (1996).

[32] H. Xi, X. Li, and J.D. Gunton, Phys. Rev. Lett. 78, 1046 (1997).

[33] V. Croquette, P. Le Gal, A. Pocheau, and R. Gugliemetti, Europhys. Lett. 1, 393 (1986).

[34] A. Pocheau, V. Croquette, P. Le Gal, and C. Poitu, Europhys. Lett. 3, 915 (1987).

[35] K.-H. Chiam, M.C. Lai, and H.S. Greenside (unpublished).

[36] P.F. Fischer, J. Comput. Phys. 133, 84 (1997).

[37] M.R. Paul, M.C. Cross, P.F. Fischer, and H.S. Greenside, Phys. Rev. Lett. 87, 154501 (2001).

[38] M.R. Paul, M.C. Cross, and P.F. Fischer, Phys. Rev. E 66, 046210 (2002).

[39] M.R. Paul, K.-H. Chiam, M.C. Cross, P.F. Fischer, and H.S. Greenside (unpublished).

[40] R.V. Cakmur, D.A. Egolf, B.B. Plapp, and E. Bodenschatz, Phys. Rev. Lett. 79, 1853 (1997).

[41] Movies can be downloaded from http://www.cmp.caltech. edu/ stchaos

[42] F.H. Busse, Rep. Prog. Phys. 41, 1929 (1978).

[43] J.C. Buell and I. Catton, Phys. Fluids 29, 23 (1986).

[44] A. Pocheau and V. Croquette, J. Phys. (Paris) 45, 35 (1984).

[45] E. Bodenschatz, W. Pesch, and G. Ahlers, Annu. Rev. Fluid Mech. 32, 709 (2000).

[46] M.C. Cross, Phys. Fluids 25, 936 (1982).

[47] S. Chandrasekhar, Hydrodynamic and Hydromagnetic Stability (Oxford University Press, Oxford, 1961).

[48] J. Wesfreid, Y. Pomeau, M. Dubois, C. Normand, and P. Bergé, J. Phys. (Paris) 39, 725 (1978). 